\title{
Polynomial Threshold Elements?
}

\author{
Jehoshua Bruck
}

It is a well known fact that the XOR function is not a linear threshold function. This is one of the reasons for the limited performance of neural networks models in which every node is a Linear Threshold (LT) element. A common solution to this problem is to consider generalized networks in which every node is performing a polynomial threshold function. A polynomial threshold (PT) element computes the sign of a polynomial (instead of a linear form in an LT element) with the restriction that the number of terms in the polynomial is "small."

In order to evaluate the idea of generalized networks I will address the following fundamental question: what is the power of a PT element with respect to LT elements? The answer is that we do not gain much by using PTs instead of LT's. A "small" two-layer network of LT's can compute strictly more than a single PT element. In order to answer this question I have developed a novel technique that is based on Harmonic Analysis and derived lower bounds on the number of terms in such a representation. I will discuss this technique and use it to exhibit a Boolean function that can be computed by a "small" two-layer network of LT's but can not be computed by a single PT element. 